\title{
Collagen fibril organization within rat vertebral bone modified with metastatic involvement
} Burke M, Golaraei A, Atkins A, Akens M, Barzda V, Whyne C
Version Post-print/Accepted Manuscript

Citation Burke M, Golaraei A, Atkins A, Akens M, Barzda V, Whyne C.

(published version) Collagen fibril organization within rat vertebral bone modified with metastatic involvement. J Struct Biol. 2017 Aug;199(2):153-164. doi: 10.1016/j.jsb.2017.06.008.

Copyright/License

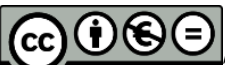

BY NC ND This work is licensed under the Creative Commons Attribution-NonCommercial-NoDerivatives 4.0 International License. To view a copy of this license, visit Creative Commons NC BY ND 4.0 License.

How to cite TSpace items

Always cite the published version, so the author(s) will receive recognition through services that track citation counts, e.g. Scopus. If you need to cite the page number of the author manuscript from TSpace because you cannot access the published version, then cite the TSpace version in addition to the published version using the permanent URI (handle) found on the record page.

This article was made openly accessible by $U$ of $T$ Faculty. Please tell us how this access benefits you. Your story matters. 


\title{
Collagen fibril organization within rat vertebral bone modified with metastatic involvement
}

Mikhail Burke a,b, Ahmad Golaraei c,d, Ayelet Atkins b, Margarete Akens e,f, Virginijus Barzda c,d, Cari Whyne a,b,e,

a Institution of Biomaterials and Biomedical Engineering, University of Toronto, Toronto, ON, Canada b Orthopaedics Biomechanics Laboratory, Sunnybrook Research Institute, Toronto, ON, Canada c Department of Physics and Institute for Optical Sciences, University of Toronto, Toronto, ON, Canada d Department of Chemical \& Physical Sciences, University of Toronto Mississauga, Mississauga, ON, Canada e Department of Surgery, University of Toronto, Toronto, ON, Canada f Techna, University Health Network, Toronto, ON, Canada

Keywords: Collagen-I, Metastasis, Vertebral bone, Transmission electron microscopy, Second harmonic generation

\begin{abstract}
:
Metastatic involvement diminishes the mechanical integrity of vertebral bone, however its specific impact on the structural characteristics of a primary constituent of bone tissue, the collagen-I fibril matrix, has not been adequately characterized. Female athymic rats were inoculated with HeLa or Ace- 1 cancer cells lines producing osteolytic or mixed (osteolytic \& osteoblastic) metastases respectively. A maximum of 21 days was allowed between inoculation and rat sacrifice for vertebrae extraction. Linear polarization-in, polarization-out (PIPO) second harmonic generation (SHG) and transmission electron microscopy (TEM) imaging was utilized to assess the impact of metastatic involvement on collagen fibril organization. Increased observations of deviations in the typical plywood motif or a parallel packing structure and an increased average measured susceptibility ratio (related to relative degree of in-plane vs. out-plane fibrils in the analyzed tissue area) in bone adjacent to metastatic involvement was indicative of change in fibrilar organization compared to healthy controls. In particular, collagen-I fibrils in tumour induced osteoblastic bone growth showed no adherence to the plywood motif or parallel packing structure seen in healthy lamellar bone, exhibiting a much higher susceptibility ratio and degree of fibril disorder. Negative correlations were established between measured susceptibility ratios and the hardness and modulus of metastatic bone tissue assessed in a previous study. Characterizing modifications in tissue level properties is key in defining bone quality in the presence of metastatic disease and their potential impact on material behaviour.
\end{abstract}

\section{Introduction}

The spread of cancer to bone most commonly affects the vertebral column, occurring in $90 \%$ of prostate, $75 \%$ of breast, $45 \%$ of

lung, and $30 \%$ of renal terminal cancer patients (Wong et al., 1990). Metastatic spread to the vertebral bodies occurs via the vertebral venous plexus leading cancer cells into a microenvironment 
within the bone that is rich in growth factors and cytokines conducive to metastatic propagation and growth (Guise, 2010; Ratliff and Cooper, 2004). The presence of tumour cells impacts the natural bone remodelling and turnover cycle causing excess bone formation (osteoblastic), bone resorption (osteolytic) or a mixture of the two. While breast and lung cancers exhibit an affinity to produce osteolytic tumours, prostate cancer yields more osteoblastic disease (Guise, 2010).

Previous studies have highlighted the impact of metastatic disease on the architecture of trabecular bone and its mineral density (Kaneko et al., 2004; Wise-Milestone et al., 2012; Nazarian et al., 2008), however less focus has been placed on potential modifications of tissue microstructure and/or nanostructure that determines the intrinsic material characteristics of the bone matrix. The formation of vertebral bone tissue matrix is completed via endochondral ossification in which a calcified cartilage is used as the base for the formation of an initial woven bone. This woven bone, with its anisotropic collagen type I (collagen-I) fibril foundation, is eventually remodelled to form mature lamellar bone, which consists of a rectilinear array of collagen-I fibrils with hydroxyapatite crystals embedded within the inter and intrafibrillar spacing (Shapiro, 2008). The presence, morphology and distribution of these constituents influence the material properties of the bone. Since the hydroxyapatite mineral is critical in providing the strength and stiffness of bone; emphasis is usually placed on the impact of metastases on the mineral content of bone tissue (Kaneko et al., 2004; Wise-Milestone et al., 2012; Nazarian et al., 2008). However, it is the rectilinear array of collagen-I fibrils that provides quasi-brittle bone with its toughness (Wang et al., 
2002). Hence, a clear understanding of the potential impact of metastatic involvement on the structure and organization of collagen fibrils is required to characterise potential changes in bone material properties.

Characterization of collagen structure within bone tissue can be performed on microstructural and nanostructural levels. The microstructural level describes the arrangement and organization of fibrils within the tissue. General fibre composite theory highlights the importance of fibre organization and alignment to a material's mechanical performance (Roylance, 2000); which could be applied microscopically to relate the alignment and organization of collagen-I fibrils and the mechanical properties of the bone tissue. This is supported by findings that microstructural changes in collagen fibril arrangement accounted for the maintenance of bone strength despite reduced mineral density in bone subjected to prolonged physical activity (Puustjarvi et al., 1999), and the relationship between orientation dependent fracture energy and collagen orientation in bone (Peterlik et al., 2006). Tissue-level assessed mechanical properties of bone tissue such as modulus and hardness have been shown to be impacted by differences in relative collagen orientation (Zysset et al., 1999; Lau et al., 2010). The nanostructural level describes the morphological and structural characteristics of a single fibril; and interactions between the neighbouring fibrils. Collagen fibril diameter has been previously described as a critical factor related to the biomechanical performance of tissues (Parry et al., 1978; Baek et al., 1998). Modifications in fibril diameter are also often associated with changes in intrafibrillar cross-linking (Christiansen et al., 2000); which in turn is linked to changes in observed bone strength and post-yield properties 
(Oxlund et al., 1995; Saito et al., 2006; Hernandez et al., 2005).

Our group has previously studied the impact of metastatic presence on the biochemical properties of collagen including hydroxylation and cross-link formation in vertebral bone. Observed changes in intrafibrilar pyridinium cross-link content, nonenzymatic cross-link formation, mature/immature cross-link ratios and hydroxylation of residues show a clear change/modification within collagen fibrils (Burke et al., 2016). Such changes have been linked to modified mechanical behaviour of bone (Saito and Marumo, 2010). However, there is still a lack of information on the impact of metastatic disease on collagen fibril organization, morphology and packing, which also contribute to the mechanical properties of bone (Martin and Boardman, 1993). Second-harmonic generation (SHG) is a coherent process where two photons of the same wavelength interact with a noncentrosymmetric material and produce a photon of half the wavelength.

The intensity and polarization of the SHG signal are highly dependent on the nanostructure and microstructural level of organization of the tissue, and can be used to describe the noncentrosymmetric organization of collagen fibrils (Roth and Freund, 1979; Freund and Deutsch, 1986). Transmission electron microscopy (TEM) can then be used in conjunction with the polarization SHG microscopy to assess the nanostructural details of collagen-I motifs and cross-sections measurements of the dimensions of the collagen-I fibrils in bone and other tissues at high magnifications (Weiner et al., 1997; Rubin et al., 2004; Shauly et al., 1992). These techniques have been utilized to assess the variations in the collagen organization, motif and/or fibril diameter within bone 
impacted by pathologic conditions such as osteogenia imperfecta and osteoporosis (Kafantari et al., 2000; Nadiarnykh et al., 2007). This study seeks to assess whether metastatic disease has a significant effect on collagen-I fibril organization and diameter in vertebral bone. The previously established modifications in crosslinking; hydroxylation and other features of collagen-I within bone due to the presence of tumour (Burke et al., 2016) and previously observed changes in fibril orientation and diameter in various bone pathologies (Kafantari et al., 2000; Nadiarnykh et al., 2007) suggest the hypothesis that modifications in both fibril diameter and organization in bone tissue may be present in bone with osteolytic and osteoblastic metastatic involvement.

\section{Material/methods}

\section{Animal model and metastatic inoculation}

Rat models utilizing systemic inoculation of human HeLa cervical cancer cells and canine Ace-1 prostate cancer cells have been shown to produce osteolytic and mixed osteolytic/osteoblastic metastatic lesions; respectively, within vertebral bone at a success rate of approximately 66\% (Wise-Milestone et al., 2012; Engebraaten and Fodstad, 1999; Won et al., 2010). These previously established models were used to simulate the physiologic development of vertebral metastases under an animal use protocol approved by the Ontario Cancer Institute. Athymic female Hsd: RHFoxn1rnu rats (5-6 weeks old) were inoculated with human HeLa cervical cancer cells (previously misidentified as MT-1 cells) to create osteolytic metastases $(N=17)$. Canine Ace-1 prostate cancer cells were similarly used to produce mixed (osteolytic/ osteoblastic) metastases ( $N=17)$. An additional 12 rats were used as healthy controls. The HeLa and ACE-1 cell lines were stably 
transfected with the luciferase gene to enable bioluminescent image monitoring of tumour growth. Intracardiac injections containing $1.5 \longrightarrow 106$ cells (in $0.2 \mathrm{~mL}$ of media) were conducted under anaesthetic (nose-cone inhalation, $2 \%$ isoflurane/air mixture). 21 days after injection, bioluminescence imaging (Xenogen) was performed for detection and semi-qualitative assessment of the degree of metastatic involvement in the spine (Wise-Milestone et al., 2012; Sadikot and Blackwell, 2005). After euthanasia (via CO2 asphyxiation), vertebrae were harvested, placed in $8 \%$ glutaraldehyde fixative solution and maintained at $4 \longrightarrow$ C. The 11 th thoracic (T11) vertebrae were decalcified in 10\% EDTA solution and cut in half along the sagittal plane, with one half utilized for polarimetric SHG analysis and the other half for TEM analysis. In cases of premature rat death, the vertebrae were removed immediately and noted accordingly.

\section{Polarimetric SHG microscopy sample preparation and data acquisition}

Linear polarization-in, polarization-out (PIPO) SHG microscopy imaging allows for specific imaging and assessment of collagen fibril organization within tissue. For linear PIPO SHG, T11 half-vertebra samples were dehydrated and paraffin embedded and $5 \mu \mathrm{m}$ sections were cut and mounted on glass slides. Sections were stained with haematoxylin and eosin (H\&E) for histopathologic analysis. Chosen regions of interest (ROI) were divided into trabecular bone that had not completed endochrondral ossification (therefore a mixture of lamellar bone, woven bone and cartilage; typically located towards the endplates) versus fully lamellar bone (typically located in the middle of the trabecular centrum). To assess the effect of tumour burden on relative collagen fibril orientation, ROIs were further divided to represent trabecular bone adjacent to tumour and trabecular bone adjacent to unaffected marrow. Additionally, due to the mixed nature of Ace-1 tumour involvement, another ROI specific only to Ace-1 inoculated samples was isolated and identified as pathological new bone formation resulting from osteoblastic lesions. All imaging results were compared to analyzed control samples. Figure 1 provides a simple schematic of the ROI breakdown.

The linear PIPO SHG microscopy imaging was conducted with a home-built laser scanning nonlinear microscope. A diode-pumped ytterbium-ion-doped potassium gadolinium tungstate ( $\mathrm{Yb}: \mathrm{KGW}$ ) crystal based laser oscillator\{\{230 Major,A. 2006\}\} was used for imaging. The microscope contained a linear polarizer to ensure that the incoming laser beam is linearly polarized. A half-wave plate (Comar Optics) was placed before the excitation objective (20x/0.75 NA air objective, Carl Zeiss) to change the orientation of linear polarization of the incoming laser beam. The emitted SHG signal from the sample 
was collected in the forward direction using a custom-designed 0.85 NA objective. A linear polarizer (Laser component Inc.) was placed after the collection objective and before the SHG detector (Hamamatsu model H7421-40) to measure the polarization of emitted SHG from the sample. A BG 39 filter and a 510-520 nm band-pass interference filter (Edmund Optics) were used to separate SHG from the laser radiation. The half-wave plate was rotated to 9 evenly spaced angles from 0 to $\pi$, while analyzer was rotated to 9 different angles with equal increments for each polarizer orientation angle to perform the linear PIPO SHG imaging. Various locations in the samples were selected and $110 \times 110 \mu \mathrm{m}$ areas were laser beam raster scanned using galvanometric scanning mirrors (Cambridge Technology).

The measured SHG intensity depends on the incoming linear polarization orientation angle $\theta$ and linear analyzer orientation angle $\varphi$ set with respect to $Z$ axis of the Cartesian laboratory coordinate system, where $X Z$ is the image plane and $Y$ is the principal beam propagation direction. The SHG intensity can be fit for each pixel of the image with the following expression\{\{169 Tuer,A.E. 2012\}\}:

$$
I_{S H G} \propto\left|\sin (\varphi-\delta) \sin 2(\theta-\delta)+\cos (\varphi-\delta) \sin ^{2}(\theta-\delta)+\frac{\chi_{Z Z Z}^{(2)},}{\chi_{Z X X}^{(2)}} \cos (\varphi-\delta) \cos ^{2}(\theta-\delta)\right|^{2} .
$$

where second-order nonlinear susceptibility component ratio $\chi_{Z Z Z}^{(2)} / \chi_{Z X X}^{(2)}{ }^{\prime}$ and the average fibril orientation $\delta$ are the fitting parameters. The nonlinear susceptibility tensor components $\chi_{z Z z}^{(2)}$ ' and $\chi_{\text {zXX }}^{(2)}$ ' are defined in the molecular projection reference frame with the effective cylindrical collagen fiber axis $z$ projected onto $X Z$ image plane. The fibril projection axis orientation ( $Z^{\prime}$ axis) is defined by the angle $\delta$ from the $Z$-laboratory axis. For the fitting analysis, the sample birefringence is neglected since the sample is $5 \mu \mathrm{m}$ thick. Kleinman symmetry is assumed because the excitation laser wavelength (1028 $\mathrm{nm}$ ) is far from the collagen absorption wavelength\{\{169 Tuer,A.E. 2012; 290 Golaraei,Ahmad 2016\}\}. A trust region reflective (TRR) fitting algorithm written in MATLAB was used to extract the fitting parameters $\chi_{Z Z Z}^{(2)} / \chi_{Z X X}^{(2)}{ }^{\prime}$ and $\delta$ were extracted for each pixel of the image.

\section{TEM sample preparation and data acquisition}

Transmission electron microscopy (TEM) enables qualitative assessment of the arrangement of collagen fibrils and quantification of collagen fibril diameter in bone tissue. For TEM; the contralateral T11 halfvertebra samples were post-fixed in osmium tetroxide, dehydrated with pure methanol and embedded into an epoxy resin. Thin sections (70-100 nm) were cut along the sagittal plane of the bone utilizing an Ultracut ultramicrotome (Leica Biosystem). An initial section was stained with toluidine blue in order to select ROls including: existing trabecular bone adjacent to tumour lesions and identified pathological new (osteoblastic) bone formation. Subsequent sections were post-stained with lead citrate and uranyl acetate in order to enhance the visibility of the fibrils and placed on carbon TEM grids for analysis.

Electron microscopy was performed on a FEI Tecnai 20 TEM operating at $200 \mathrm{kV}$ with micrographs obtained between $6500 x$ and 50,000x magnification. Electron micrographs between 6500x and 25,000x were utilized for qualitative analysis on collagen fibril motif and organization. Micrographs between 25,000x and 50,000x were utilized for quantitative analysis.

To assess the orientation of longitudinally oriented collagen fibrils, a computed histogram distribution analysis was performed via the "directionality" plugin (ImageJ/FIJI) as established in other studies 
(Reznikov et al., 2015; Atkins et al., 2015). Multiple ROIs for analysis were selected from 2 to 3 micrographs per rat and the standard deviation of the observed Gaussian distribution of angular orientation of the fibrils is indicative of the degree of orientation disorder relative to the identified peak describing the preferred orientation. This parameter describes the relative degree of orientation disorder within areas of lamellar bone or pathological new bone growth.

Micrographs containing axial cross-sections of collagen fibrils were image processed (i.e. normalized local contrast enhancement, despeckling), binarized and the diameter of fibrils measured via the "analyze particle" script (ImageJ/FIJI). The minimal Feret diameter measurement was taken to account for deviations in perpendicular cut and an average of 500 fibrils were measured per rat to determine average fibril diameter for each sample group.

\section{Statistical analysis}

The normality of each dataset was evaluated utilizing the Shapiro-Wilk test. For PIPO SHG data analysis, a multiple-way ANOVA was then performed based on a parametric mixed model. The fixed effects were "Metastatic Involvement" (Ace-1, HeLa and Healthy), "Bone Tissue State" (fully lamellar bone (L), bone exhibiting incomplete ossification (IO) and pathologic new bone (New)) and "Adjacent to Tumour" (Yes

or No). As no significant interaction effect was seen between the variables of "Bone Tissue State", "Metastatic Involvement" or "Adjacent to Tumour", Tukey post hoc tests were used to assess differences between groups. For TEM data analysis where only one factor varied ("Metastatic Involvement"), Welsh followed by Tamhane post hoc tests for multiple comparisons were performed to test for differences between sample groups without equal variance. In cases with equal variance and normality, analysis of variance (ANOVA) followed by Tukey post hoc test was used. In all cases, data was presented as mean \pm standard error, with a significance level of $p<0.05$ (SPSS v23, SPSS, Chicago, IL, USA).

Additionally, simple linear regression models (equivalent to bivariate analysis) were made between susceptibility ratio measurements and nanoindentation data from equivalent ROI from our previous work (Burke et al., 2017) in order to establish the potential influence of collagen organization on nanomechanical behaviour.

\section{Results}

\section{Sample characterization}

Bioluminescent imaging showed metastatic tumour growth in the spine in 14/17 rats injected with HeLa cells and 12/17 rats injected with Ace-1 cells (Fig. 2). Additional tumour signal appeared in the heart, lungs and femurs. H\&E stained sections of the T11 vertebrae (Fig. 3a-C) showed established tumour burden (tumour cells distinguishable from healthy marrow cells due to larger cell size inferred by nuclei spacing and/or presence of stroma - Fig. $3 b$ and c) in 10/17 rats injected with HeLa and 8/17 rats injected with Ace-1 cells. A total of 30 rats (HeLa: $N=10$; Ace-1: $N=8$; Healthy: $N=12$ ) were utilized for PIPO SHG analysis. For TEM imaging, 13 rats (HeLa: $N=5$; Ace-1: $N=3$; Healthy: $N=5$ ) were utilized for fibril diameter measurement and 14 rats (HeLa: $N=5$; Ace-1: $N=3$; Healthy: $N=6$ ) were utilized for directionality analysis.

\section{PIPO SHG microscopy}


PIPO SHG microscopy imaging results of trabeculae in healthy control rats, HeLa cervical cancer cellinjected rats and Ace- 1 prostate cancer cell-injected rats are shown in Figs. 4-6 respectively. In each figure, the first column shows the H\&E image where the scanned area is indicated by the black square. The second column presents SHG intensity images of the scanned areas for each case. The nonlinear susceptibility component ratio $\chi_{Z Z Z}^{(2) '} / \chi_{Z X X}^{(2)}$ for each pixel of the image is extracted and displayed as a color-coded image shown in the third column of each figure. The occurrence frequency histograms of the susceptibility tensor component ratio for all pixels in the images are shown in the last column. The mean susceptibility component ratio values extracted from the histograms of each bone tissue type and metastatic involvement group of scanned areas are presented in Table 1.

A multilamellar feature with alternating bright and dark regions can be seen in the control bone in Fig. $4 \mathrm{~d}$. These structures are predominantly present in the lamellar bone and imply a plywood organization of collagen in the trabeculae. The nonlinear susceptibility ratio depends on the orientation of collagen fibrils as well as its intrinsic structural properties). Therefore, the regions of lower susceptibility ratio values indicate that fibrils are oriented closer to the image plane, while higher ratio corresponds to the larger out-of-plane fibril orientation angle. For the trabeculae areas with incomplete ossification, the irregular "speckle" patterns of the susceptibility ratio and SHG intensity can be observed, while the lamellar domains are less common (Fig. 4c).

The susceptibility ratio showed a slight but significant increase $(\sim 1.5 \%, p<0.05)$ in bone near the endplates with incomplete ossification compared to fully lamellar bone (Table 1a) with the ratio in the "speckle" regions appearing to be higher and not correlated with SHG intensity. Observed differences in susceptibility ratio between bone from Ace- 1 or HeLa inoculated rats and healthy controls ( $2.4 \%$ and $\sim 1.3 \%$ respectively) showed no statistical significance when measured globally (Table $1 \mathrm{~b}$ ). However, there was a statistically significant increase $(\sim 2.8 \%, p<0.05)$ in susceptibility ratio with bone adjacent to tumour compared to bone adjacent to marrow (Table 1c). This suggests a lack of difference between bone not adjacent to tumour (i.e. surrounded by marrow) from tumour inoculated specimens and bone from healthy controls indicating the importance of tumour proximity in the discussion of any observed modifications in bone samples. New osteoblastic bone growth within Ace-1 samples showed the greatest increase in susceptibility ratio (from $3-4.6 \%, p<0.05$ ) compared to bone from other locations (Table 1a) indicating a high degree of disorder and/or lack of in-plane alignment. Negative correlations were established between the susceptibility ratios measured and the modulus and hardness of metastatic bone tissue (Burke et al., 2017) (modulus: R2 $=0.374$ and for hardness: R2 $=0.502$ ).

\section{Transmission electron microscopy}

TEM images confirmed a lamellar bone structure in all 3 sample groups exhibiting the characteristic plywood motif (Fig. 7a-c). The plywood motif appeared twisted in nature, showing a transition from orthogonal to oblique to longitudinal fibrils, or orthogonal with direct transition from orthogonal to longitudinal fibrils. There were also regions within lamellar bone in all 3 sample groups, which deviated from the plywood motif (Fig. 8a-c) typically adjacent to woven bone or cartilage (indicative of nascent lamellar bone). In particular, osteoblastic bone in the Ace-1 model exhibited no consistent motif and was highly disorganized (Fig. 8a) compared to lamellar bone. This pathological new bone growth showed different characteristics in comparison to healthy woven bone. For example healthy woven bone, present in trabeculae with incomplete endochondral ossification by the caudal and cranial ends of the 
vertebral body, exhibited a dense concentration of canaliculi not seen in the pathologic new bone (Fig. 9b).

Results of TEM image assessment via directionality analysis showed clear delineation between parallel packed fibrils and disorganized fibrils as shown in Fig. 10. The degree of disorder for fibrils oriented longitudinally relative to the observation plane was modified with Ace-1 tumour involvement. Both older osteolytic bone adjacent to Ace-1 tumour tissue and osteoblastic bone showed significant increases $(23.7 \%$ and $28.2 \%$ respectively, $p<0.05)$ in the degree of disorder in fibril orientation compared to healthy controls. There was no significant difference in disorder observed between bone adjacent to HeLa tumour tissue and healthy controls (Fig. 11).

Although the mean fibril diameter was slightly smaller in healthy controls compared to tumour bearing samples $(\sim 4.7 \%)$ there was no significant difference in measured fibril diameters between sample groups (Table 2).

\section{Discussion}

Both osteolytic and mixed metastatic involvement were shown to impact collagen fibril organization within vertebral trabecular bone with increased deviations within the plywood motif commonly associated with lamellar bone. In particular, observed pathological osteoblastic new bone growth specific to Ace-1 metastatic involvement, showed a highly disorganized woven bone matrix. The structural modifications of collagen were deduced using comparative studies of polarimetric SHG microscopy and transmission electron microscopy. The PIPO SHG microscopy utilized in analysis of the collagen fibril network in the bone tissue is sensitive to the hierarchical structure of collagen starting from the amino acid organization in the triple helix of collagen and arrangement of triple helixes in the fibrils as well as fibril organization within the focal volume (voxel) of the tissue. Therefore, the information obtained from the TEM images about the organization of collagen fibrils in the tissue can help to unravel the origin of SHG signal changes that depends on the internal structure of the fibrils and also on the changes in the fibril alignment within the microscopic focal volume (voxel) of the tissue.

Endochondral ossification leads to a lamellar bone structure whose collagen fibril matrix displays a plywood motif consisting of layers of parallel packed collagen fibrils (Weiner et al., 1997). Normal bone remodelling occurs via the formation of multiple regions (or "pockets") of focal resorption and subsequent bone creation that maintains this lamellar structure (Clarke, 2008). Unlike the rod and plate structure of normal trabecular bone, the Ace-1 new bone formation yielded a "feathery/lattice" structure. SHG imaging of this pathological new bone showed an increase in the susceptibility ratio which may be indicative of enhanced disorganization of the collagen fibrils within the tissue (Tuer et al., 2012) and TEM imaging confirmed that this osteoblastic bone had a woven bone structure with a higher degree of disorder in contrast to normal lamellar structure. Typically, the production of woven bone from osteoblasts can be expected in the initial stages of endochondral ossification or in fracture healing (Shapiro, 2008). However, the formation of woven bone in lieu of lamellar bone on existing nonfractured lamellar bone highlights the modified operation of osteoblasts within the metastatic environment. Woven bone in general is known to have weaker mechanical properties than its lamellar counterparts (Clarke, 2008) and as such its increased presence could impact the mechanical integrity of the vertebral structure. 
Interestingly, while the woven bone tissue structure of this osteoblastic new bone could be indicative of it nascent nature, potential tissue pathology was highlighted by the qualitatively observed decrease in the concentration of canaliculi between osteoblastic tissue (metastatically-induced woven bone) relative to healthy woven bone produced during endochondral ossification (Fig. 8). The lacunar-canalicular system (LCS) in bone tissue may impact the ability of osteocytes to sense mechanical loading (important for remodelling and bone volume), to communicate with other cells in the microenvironment and to obtain nutrients (Lai et al., 2015; Kobayashi et al., 2015; Sharma et al., 2012). Therefore, the reduced number of observed canaliculi with metastatic involvement compared to healthy woven bone could impact subsequent remodelling and hence the quality of the bone, as shown in other pathologic conditions (Kobayashi et al., 2015). The presence of these observed bone tissue abnormalities with osteoblastic new bone suggest a modification in osteoblast function in the presence of metastases. It has been suggested that the mesh-like membrane morphology of osteoblasts could impact the fibril organization of formed bone osteoid (Pazzaglia et al., 2010) and that pro-osteoblastic factors such as transforming growth factor-beta (TGF-b), associated with prostate cancer-induced bone metastasis (Mishra et al., 2011), can influence the morphology of osteoblasts with metastatic involvement (Mercer and Mastro, 2005). Such a change in osteoblast membrane morphology could then potentially explain the observed modification of canaliculi concentration and fibril organization within this pathological osteoblastic bone via TEM imaging.

Although the differences in collagen fibril organization within pathological osteoblastic bone were profound, increases in susceptibility ratio in identified osteolytic bone adjacent to tumour also suggest a potential modification in collagen fibril organization. TEM analysis showed that while such bone often exhibited a lamellar bone structure (Fig. 6) that increased deviations from the plywood motif also occurred (Fig. 7a and b). These deviations, combined with directionality analysis of in-plane fibrils showing an increase in the degree of disorder of osteolytic bone adjacent to Ace-1 tumour, confirmed greater fibril disorganization as suggested by the increased susceptibility ratio seen in SHG imaging. This quantified disorganization of collagen fibrils in the bone adjacent to cancer cells could be due to modifications in the bone microenvironment. Potential changes in acidity (Yoneda et al., 2011), small leucine-rich proteoglycans (SLRPs) concentration (Bi and Yang, 2013; Weber et al., 2001), fibrilassociated collagen with interrupted triple helices' (FACITs) concentration (Yen et al., 2014) and crosslink concentration (Burke et al., 2016) with metastatic involvement all could impact collagen fibrillogenesis, fibril-fibril interactions and fibril organization (Li et al., 2009; Nikitovic et al., 2012; Nishiyama et al., 1994; Saito and Marumo, 2010) leading to increased fibril disorganization. Additionally, the observed increase in susceptibility ratio in incompletely ossified bone compared to fully lamellar bone could be attributed to the healthy woven bone zone observed in TEM images (Fig. 8b). This naturally occurring woven bone likely corresponds to the observed "speckle" regions of SHG intensity observed in incompletely ossified bone. The moderate negative correlation of susceptibility ratio to hardness and modulus data suggests that collagen organization can influence the nanomechanical behaviour of bone tissue. This maybe explained as the deformation mechanism for bone tissue is predicated in part by interfibril interactions (Zimmermann et al., 2015) which could be modified by changes in structural organization. In addition to the susceptibility ratio, PIPO SHG imaging can produce another fitting parameter: $d$. The fitting parameter $d$ provides the effective orientation angle of collagen fibrils in the image plane. The orientation distribution of collagen fibrils in the selected regions of the tissues showed no statistically significant variations except for the new bone formed in the Ace1- 
injected samples which are visible from the white light image of the H\&E samples. Hence, we focused on the variation of susceptibility ratio values.

Finally, although modifications in collagen fibril diameter has been associated with other pathologic bone conditions (Kafantari et al., 2000; Kounadi et al., 1998); no significant difference was observed in fibril diameter between sample groups in this study. This however may be due to the potential of metastatic involvement to influence a number of competing factors that can modify fibril diameter. For example, a decrease in lysine based collagen cross-linkage can lead to an increase fibril diameter (Christiansen et al., 2000). While enzymatic lysine based cross-links may decrease with metastatic involvement, non-enzymatic lysine based cross-links may increase (Burke et al., 2016). Also reductions in SLRPs such as decorin can cause a decrease in the collagen-I fibril diameter of bone tissue, whereas biglycan may increase the fibril diameter (Corsi et al., 2002). Metastatic involvement has been shown to increase the production of both of these SLRPs (Weber et al., 2001; Bi and Yang, 2013) which may have competing influences on fibril diameter.

While the results of this study show clear changes in the organization of the collagen fibril matrix of both remodelled bone adjacent to tumour and osteoblastic bone growth, some limitations must be noted with respect to the approach utilized. The tumour cell lines utilized were from different animal and tissue sources, but the immune compromised nature of the rats should minimize any impact of cell species differences in the study. However, the immunodeficient animal model may exhibit an altered inflammatory state, impacting the expression of various cytokines which are noted signalling factors within the bone remodelling process (Deshpande et al., 2013). Yet, as the healthy control model was also immunodeficient in nature, the inflammatory state within the animal was controlled for, suggesting differences in collagen organization and bone remodelling seen between metastatic and healthy groups should be due to metastatic involvement. The impact of metastatic involvement on bone collagen organization may also differ in the athymic animals compared to a normal, immunocompetent model. Therefore, while the influence of metastases on bone collagen organization within this athymic rat model is clear, future work investigating collagen fibril organization utilizing immunocompetent animal models could remove any potential influencing factors. The susceptibility ratio for the collagen is a function of three hierarchy levels in the collagen: The difference in the amino acid composition of the collagen, how the triple helices are assembled into collagen fibrils and the 3D distribution of collagen fibrils in the bone tissue. Modifications in the fibril organization and orientation relative to other fibrils were considered the main contributor in the observed differences in susceptibility ratio as confirmed by TEM; the potential impact of changes in amino acid organization with the triple helix of collagen or the triple helix orientation within the fibrils in the bone tissue was not investigated in this study. Also, while H\&E stained slides created for tissue differentiation and ROI identification/selection have been successfully SHG imaged in previous work (Golaraei et al., 2014; Tokarz et al., 2015; Tuer et al., 2010) it has been reported that tissue fixation and embedding may create artifacts in collagen structure (Perry et al., 2012). However, comparing the susceptibility ratio between groups (rather than absolute values of individual tensor components) may mitigate the effect of possible artifacts. Due to process and sectioning differences between the two halves of each vertebrae, a correlative mapping approach to analyze the identical area with SHG and TEM was not possible. As such, direct correlation between susceptibility ratio and degree of disorder could not be drawn.

\section{Conclusion}


This study showed that metastatic involvement influences the formation and organization of collagen fibrils within the vertebral bone matrix. Future work will be required to establish the potential impact of fibril disorder and disorganization on material and mechanical level behaviour of metastatically involved vertebral bone. Ultimately, by quantifying the influence of cancer on bone tissue, a fundamental basis for understanding the mechanics of structural changes can be established; which will be a key in developing new integrative strategies to mechanically model metastatically involved vertebral bone and highlight areas of focus in the evaluation of new and existing treatment options.

\section{Conflict of interest}

The authors declare that there are no conflicts of interest.

\section{Acknowledgements}

Grant funding for this study was provided by the Canadian Institutes of Health Research (\#68911 and \#125886).

\section{References}

Atkins, A., Reznikov, N., Ofer, L., Masic, A., Weiner, S., Shahar, R., 2015. The threedimensional structure of anosteocytic lamellated bone of fish. Acta Biomater.

13, 311-323.

Baek, G.H., Carlin, G.J., Vogrin, T.M., Woo, S.L., Harner, C.D., 1998. Quantitative analysis of collagen fibrils of human cruciate and meniscofemoral ligaments.

Clin. Orthop. Relat. Res. 357, 205-211.

$\mathrm{Bi}$, X.L., Yang, W., 2013. Biological functions of decorin in cancer. Chin. J. Cancer 32, 266-269.

Burke, M., Atkins, A., Akens, M., Willett, T., Whyne, C., 2016. Osteolytic and mixed cancer metastasis modulates collagen and mineral parameters within rat vertebral bone matrix. J. Orthop. Res.

Burke, M., Atkins, A., Kiss, A., Akens, M., Yee, A., Whyne, C., 2017. The impact of metastasis on the mineral phase of vertebral bone tissue. J. Mech. Behav.

Biomed. Mater. 69, 75.

Christiansen, D.L., Huang, E.K., Silver, F.H., 2000. Assembly of type I collagen: fusion of fibril subunits and the influence of fibril diameter on mechanical properties.

Matrix Biol. 19, 409-420.

Clarke, B., 2008. Normal bone anatomy and physiology. Clin. J. Am. Soc. Nephrol. 3

(Suppl. 3), S131-S139.

Corsi, A., Xu, T., Chen, X.D., Boyde, A., Liang, J., Mankani, M., Sommer, B., lozzo, R.V., Eichstetter, I., Robey, P.G., Bianco, P., Young, M.F., 2002. Phenotypic effects of biglycan deficiency are linked to collagen fibril abnormalities, are synergized by decorin deficiency, and mimic Ehlers-Danlos-like changes in bone and other connective tissues. J. Bone Miner. Res. 17, 1180-1189.

Deshpande, S.S., Weiss, D.M., Donneys, A., Gallagher, K.K., Tchanque-Fossuo, C.N., Sarhaddi, D., Buchman, S.R., 2013. An isogenic model of murine mandibular distraction osteogenesis. J. Craniofac. Surg. 24, 540-544.

Engebraaten, O., Fodstad, $\varnothing$., 1999. Site-specific experimental metastasis patterns of two human breast cancer cell lines in nude rats. Int. J. Cancer 82, 219-225. 
Freund, I., Deutsch, M., 1986. Second-harmonic microscopy of biological tissue. Opt. Lett. 11, 94-96.

Golaraei, A., Cisek, R., Krouglov, S., Navab, R., Niu, C., Sakashita, S., Yasufuku, K., Tsao, M.S., Wilson, B.C., Barzda, V., 2014. Characterization of collagen in non-small cell lung carcinoma with second harmonic polarization microscopy. Biomed.

Opt. Express 5, 3562-3567.

Golaraei, A., Kontenis, L., Cisek, R., Tokarz, D., Done, S.J., Wilson, B.C., Barzda, V., 2016. Changes of collagen ultrastructure in breast cancer tissue determined by second-harmonic generation double Stokes-Mueller polarimetric microscopy. Biomed. Opt. Express 7, 4054-4068.

Guise, T., 2010. Examining the metastatic niche: targeting the microenvironment. Semin. Oncol. 37 (Suppl. 2), S2-S14.

Hernandez, C.J., Tang, S.Y., Baumbach, B.M., Hwu, P.B., Sakkee, A.N., van der Ham, F., DeGroot, J., Bank, R.A., Keaveny, T.M., 2005. Trabecular microfracture and the influence of pyridinium and non-enzymatic glycation-mediated collagen crosslinks.

Bone 37, 825-832.

Kafantari, H., Kounadi, E., Fatouros, M., Milonakis, M., Tzaphlidou, M., 2000.

Structural alterations in rat skin and bone collagen fibrils induced by ovariectomy. Bone 26, 349-353.

Kaneko, T.S., Bell, J.S., Pejcic, M.R., Tehranzadeh, J., Keyak, J.H., 2004. Mechanical properties, density and quantitative CT scan data of trabecular bone with and without metastases. J. Biomech. 37, 523-530.

Kobayashi, K., Nojiri, H., Saita, Y., Morikawa, D., Ozawa, Y., Watanabe, K., Koike, M., Asou, Y., Shirasawa, T., Yokote, K., Kaneko, K., Shimizu, T., 2015. Mitochondrial superoxide in osteocytes perturbs canalicular networks in the setting of agerelated osteoporosis. Sci. Rep. 5, 9148.

Kounadi, E., Fountos, G., Tzaphlidou, M., 1998. The influence of inflammationmediated osteopenia (IMO) on the structure of rabbit bone and skin collagen

fibrils. Connect Tissue Res. 37, 69-76.

Lai, X., Price, C., Modla, S., Thompson, W.R., Caplan, J., Kirn-Safran, C., Wang, L., 2015. The dependences of osteocyte network on bone compartment, age, and disease.

Bone Res. 3, 15009.

Lau, M., Lau, K., Yao Yeo, Y., Au Yeung, C., Lee, J., 2010. Measurement of bovine bone properties through surface indentation technique. Mater. Manuf. Processes 25,

324-328.

Li, Y., Asadi, A., Monroe, M.R., Douglas, E.P., 2009. PH effects on collagen fibrillogenesis in vitro: electrostatic interactions and phosphate binding.

Mater. Sci. Eng. C 29, 1643-1649.

Major, A., Cisek, R., Barzda, V., 2006. Femtosecond Yb:KGd(WO(4))(2) laser oscillator pumped by a high power fiber-coupled diode laser module. Opt.

Express 14, 12163-12168.

Martin, R.B., Boardman, D.L., 1993. The effects of collagen fiber orientation, porosity, density, and mineralization on bovine cortical bone bending properties. J.

Biomech. 26, 1047-1054. 
Mercer, R.R., Mastro, A.M., 2005. Cytokines secreted by bone-metastatic breast cancer cells alter the expression pattern of $f$-actin and reduce focal adhesion plaques in osteoblasts through PI3K. Exp. Cell Res. 310, 270-281.

Mishra, S., Tang, Y., Wang, L., deGraffenried, L., Yeh, I.T., Werner, S., Troyer, D., Copland, J.A., Sun, L.Z., 2011. Blockade of transforming growth factor-beta (TGFî2) signaling inhibits osteoblastic tumorigenesis by a novel human prostate cancer cell line. Prostate 71, 1441-1454.

Nadiarnykh, O., Plotnikov, S., Mohler, W.A., Kalajzic, I., Redford-Badwal, D., Campagnola, P.J., 2007. Second harmonic generation imaging microscopy studies of osteogenesis imperfecta. J. Biomed. Opt. 12, 051805.

Nazarian, A., von Stechow, D., Zurakowski, D., Muller, R., Snyder, B.D., 2008. Bone volume fraction explains the variation in strength and stiffness of cancellous bone affected by metastatic cancer and osteoporosis. Calcif. Tissue Int. 83, 368379.

Nikitovic, D., Aggelidakis, J., Young, M.F., lozzo, R.V., Karamanos, N.K., Tzanakakis, G. N., 2012. The biology of small leucine-rich proteoglycans in bone pathophysiology. J. Biol. Chem. 287, 33926-33933.

Nishiyama, T., McDonough, A.M., Bruns, R.R., Burgeson, R.E., 1994. Type XII and XIV collagens mediate interactions between banded collagen fibers in vitro and may modulate extracellular matrix deformability. J. Biol. Chem. 269, 28193-28199.

Oxlund, H., Barckman, M., Ortoft, G., Andreassen, T.T., 1995. Reduced concentrations of collagen cross-links are associated with reduced strength of bone. Bone 17, 365S-371S.

Parry, D.A.D., Barnes, G.R.G., Craig, A.S., 1978. A comparison of the size distribution of collagen fibrils in connective tissues as a function of age and a possible relation between fibril size distribution and mechanical properties. Proc. R. Soc. Lond. Ser. B 203, 305.

Pazzaglia, U.E., Congiu, T., Marchese, M., Dell'Orbo, C., 2010. The shape modulation of osteoblast-osteocyte transformation and its correlation with the fibrillar organization in secondary osteons: a SEM study employing the graded osmic maceration technique. Cell Tissue Res. 340, 533-540.

Perry, S.W., Burke, R.M., Brown, E.B., 2012. Two-photon and second harmonic microscopy in clinical and translational cancer research. Ann. Biomed. Eng. 40, 277-291.

Peterlik, H., Roschger, P., Klaushofer, K., Fratzl, P., 2006. From brittle to ductile fracture of bone. Nat. Mater. 5, 52-55.

Plotnikov, S.V., Millard, A.C., Campagnola, P.J., Mohler, W.A., 2006. Characterization of the myosin-based source for second-harmonic generation from muscle sarcomeres. Biophys. J. 90, 693-703.

Puustjarvi, K., Nieminen, J., Rasanen, T., Hyttinen, M., Helminen, H.J., Kroger, H., Huuskonen, J., Alhava, E., Kovanen, V., 1999. Do more highly organized collagen fibrils increase bone mechanical strength in loss of mineral density after oneyear running training? J. Bone Miner. Res. 14, 321-329.

Ratliff, J.K., Cooper, P.R., 2004. Metastatic spine tumors. South. Med. J. 97, 246-253. 
Reznikov, N., Chase, H., Brumfeld, V., Shahar, R., Weiner, S., 2015. The 3D structure of the collagen fibril network in human trabecular bone: relation to trabecular organization. Bone 71, 189-195.

Roth, S., Freund, I., 1979. Second harmonic generation in collagen. J. Chem. Phys. 70, 1637-1643.

Roylance, D., 2000. Laminated Composite Plates. Massachusetts Institute of Technology, Cambridge.

Rubin, M.A., Rubin, J., Jasiuk, I., 2004. SEM and TEM study of the hierarchical structure of $\mathrm{C} 57 \mathrm{BL} / 6 \mathrm{~J}$ and $\mathrm{C} 3 \mathrm{H} / \mathrm{HeJ}$ mice trabecular bone. Bone 35, 11-20.

Sadikot, R.T., Blackwell, T.S., 2005. Bioluminescence Imaging. Proc. Am. Thoracic Soc. 2, 537-540.

Saito, M., Marumo, K., 2010. Collagen cross-links as a determinant of bone quality: a possible explanation for bone fragility in aging, osteoporosis, and diabetes mellitus. Osteoporos. Int. 21, 195-214.

Saito, M., Fujii, K., Mori, Y., Marumo, K., 2006. Role of collagen enzymatic and glycation induced cross-links as a determinant of bone quality in spontaneously diabetic WBN/Kob rats. Osteoporos. Int. 17, 1514-1523.

Shapiro, F., 2008. Bone development and its relation to fracture repair. The role of mesenchymal osteoblasts and surface osteoblasts. Eur. Cell Mater.

$15,53-76$.

Sharma, D., Ciani, C., Ramirez Marin, P.A., Levy, J.D., Doty, S.B., Fritton, S.P., 2012.

Alterations in the osteocyte lacunar-canalicular microenvironment due to estrogen deficiency. Bone 51, 488-497.

Shauly, Y., Miller, B., Lichtig, C., Modan, M., Meyer, E., 1992. Tenon's capsule: ultrastructure of collagen fibrils in normals and infantile esotropia. Invest.

Ophthalmol. Vis. Sci. 33, 651-656.

Stoller, P., Celliers, P.M., Reiser, K.M., Rubenchik, A.M., 2003. Quantitative secondharmonic generation microscopy in collagen. Appl. Opt. 42, 5209-5219.

Tokarz, D., Cisek, R., Golaraei, A., Asa, S.L., Barzda, V., Wilson, B.C., 2015.

Ultrastructural features of collagen in thyroid carcinoma tissue observed by polarization second harmonic generation microscopy. Biomed. Opt. Express 6, 3475-3481.

Tuer, A., Tokarz, D., Prent, N., Cisek, R., Alami, J., Dumont, D.J., Bakueva, L., Rowlands, J., Barzda, V., 2010. Nonlinear multicontrast microscopy of hematoxylin-andeosinstained histological sections. J. Biomed. Opt. 15, 026018.

Tuer, A.E., Akens, M.K., Krouglov, S., Sandkuijl, D., Wilson, B.C., Whyne, C.M., Barzda, V., 2012. Hierarchical model of fibrillar collagen organization for interpreting the second-order susceptibility tensors in biological tissue. Biophys. J. 103, 2093-2105.

Wang, X., Shen, X., Li, X., Agrawal, C.M., 2002. Age-related changes in the collagen network and toughness of bone. Bone 31, 1-7.

Weber, C.K., Sommer, G., Michl, P., Fensterer, H., Weimer, M., Gansauge, F., Leder, G., Adler, G., Gress, T.M., 2001. Biglycan is overexpressed in pancreatic cancer and induces G1-arrest in pancreatic cancer cell lines. Gastroenterology 121, 657- 
667. 


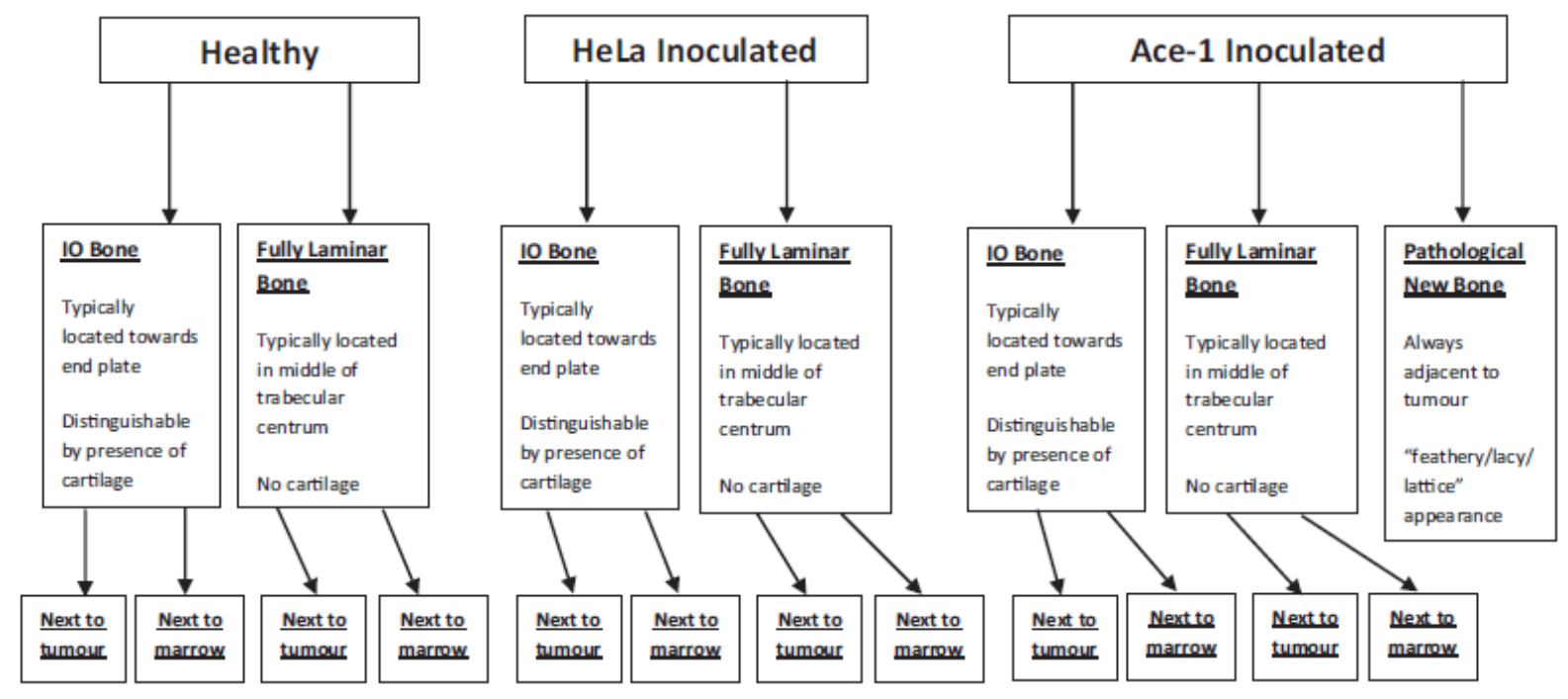

Figure 1: Simple schematic of ROI selection for SHG imaging.

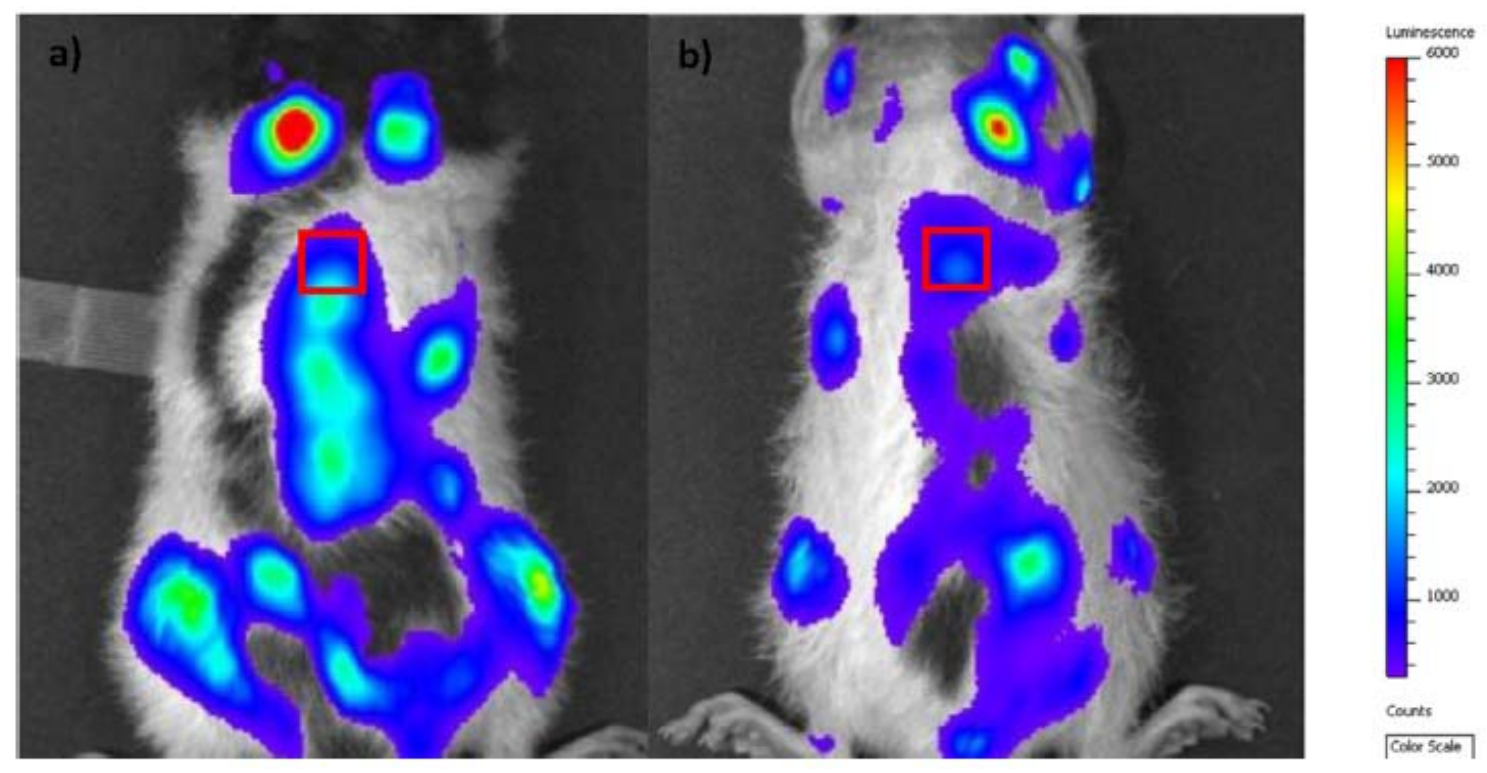

Figure 2: Representative bioluminescent images of rats inoculated with HeLa (a) and Ace-1 (b) 21 days post-inoculation. Metastatic growth was observed in various regions of the rat anatomy including the spinal column. The square overlay indicates the approximate location of T11 vertebrae. 

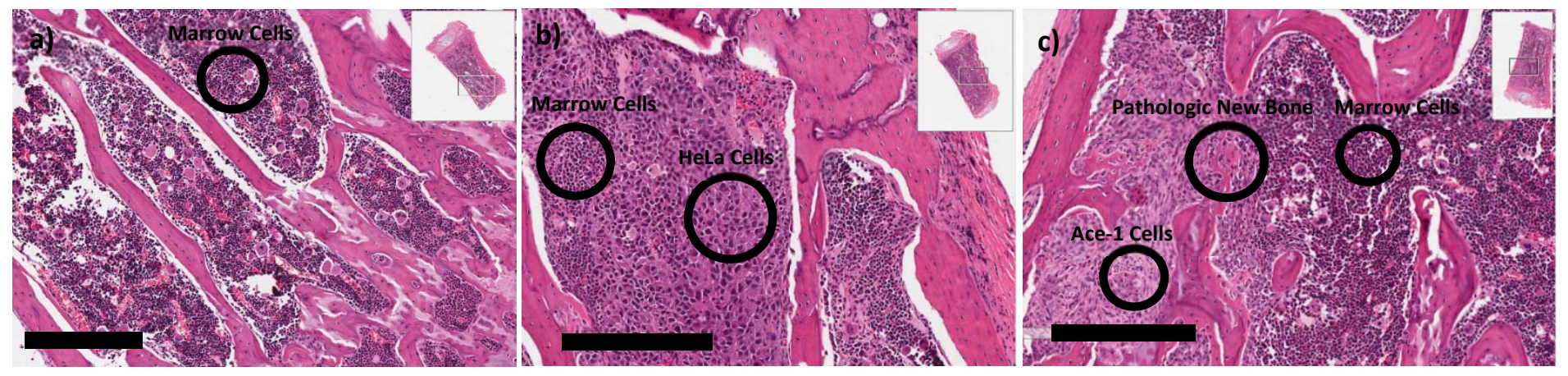

Figure 3: a) Representative H\&E stained section of T11 vertebrae of healthy control. b) Representative H\&E stained section of T11 vertebrae with HeLa tumour growth. c) Representative H\&E stained section of T11 vertebrae with Ace-1 tumour growth. Pathological new bone growth is observed. Size and morphology distinction between marrow cells and infiltrative HeLa and Ace-1 cells is also apparent. Scale bar 300um.
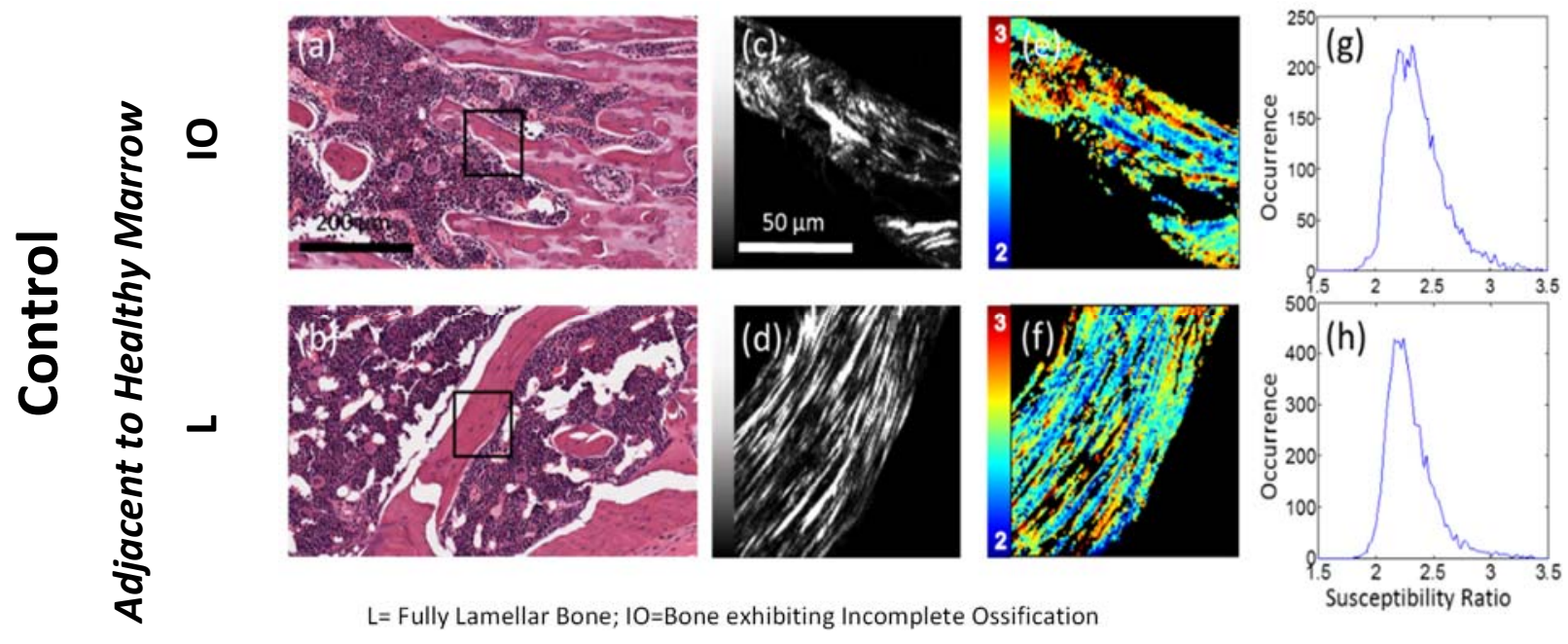

$\mathrm{L}=$ Fully Lamellar Bone; $1 \mathrm{O}=$ Bone exhibiting Incomplete Ossification

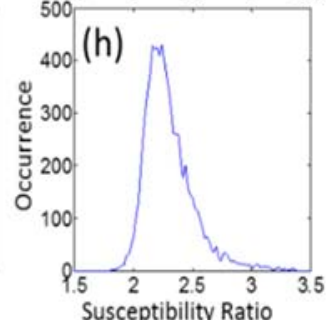

Figure 4: Representative PIPO analysis of trabeculae in healthy control rats. Panels (a-b) show bright field microscopy images of the H\&E stained tissues. Panels (cd) demonstrate the SHG intensity images (scaled for visibility) of the corresponding region outlined as a black box in H\&E images where the black box represents an area of $110 \mu \mathrm{m} \times 110 \mu \mathrm{m}$ and the scale bar in the SHG images represents $50 \mu \mathrm{m}$. Panels (e-f) display the susceptibility ratio map where the blue color represents a susceptibility ratio of 2 and the red color represents a susceptibility ratio of 3 . Panel (g-h) shows the susceptibility ratio occurrence histogram of corresponding 

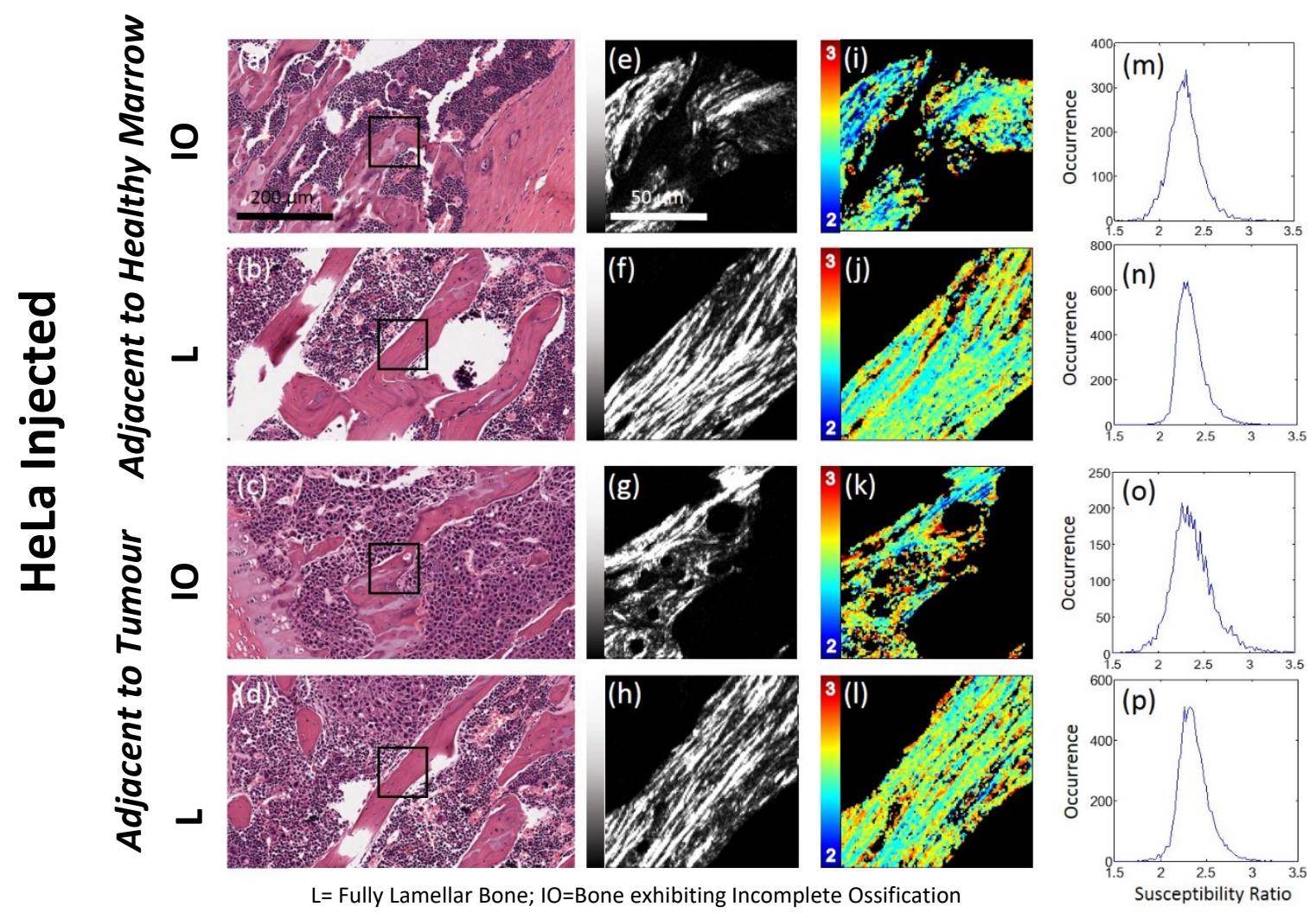

Figure 5: Representative PIPO analysis of trabeculae in HeLa cervical cancer cells injected rats. Panels (a-d) show bright field microscopy images of the H\&E stained tissues. Panels (e-h) demonstrate the SHG intensity images (scaled for visibility) of the corresponding region outlined as a black box in H\&E images where the black box represents an area of $110 \mu \mathrm{m} \times 110 \mu \mathrm{m}$ and the scale bar in the SHG images represents $50 \mu \mathrm{m}$. Panels (i-I) display the susceptibility ratio map where the blue color represents a susceptibility ratio of 2 and the red color represents a susceptibility ratio of 3 . Panel $(m-p)$ shows the susceptibility ratio occurrence histogram of corresponding pixels in panel (i-I). 

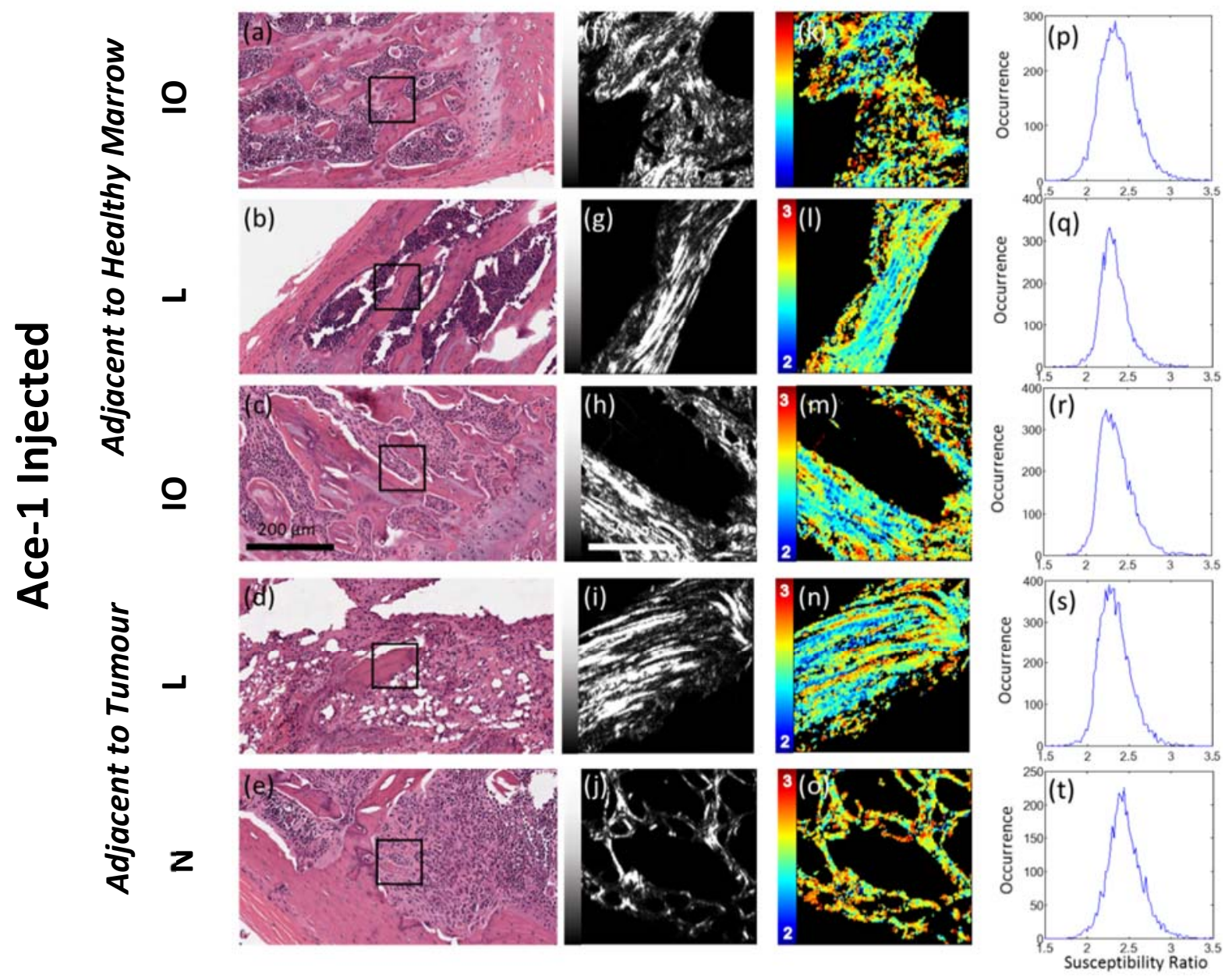

L= Fully Lamellar Bone; IO=Bone exhibiting Incomplete Ossification; N= New Bone

Figure 6: Representative PIPO analysis of trabeculae in Ace-1 prostate cancer cells injected rats. Panels (a-e) show bright field microscopy images of the H\&E stained tissues. Panels $(f-j)$ demonstrate the SHG intensity images (scaled for visibility) of the corresponding region outlined as a black box in H\&E images where the black box represents an area of $110 \mu \mathrm{m} \times 110 \mu \mathrm{m}$ and the scale bar in the SHG images represents $50 \mu \mathrm{m}$. Panels (k-o) display the susceptibility ratio map where the blue color represents a susceptibility ratio of 2 and the red color represents a susceptibility ratio of 3 . Panel ( $p-t)$ shows the susceptibility ratio occurrence histogram of corresponding pixels in panel ( $k-0)$. 

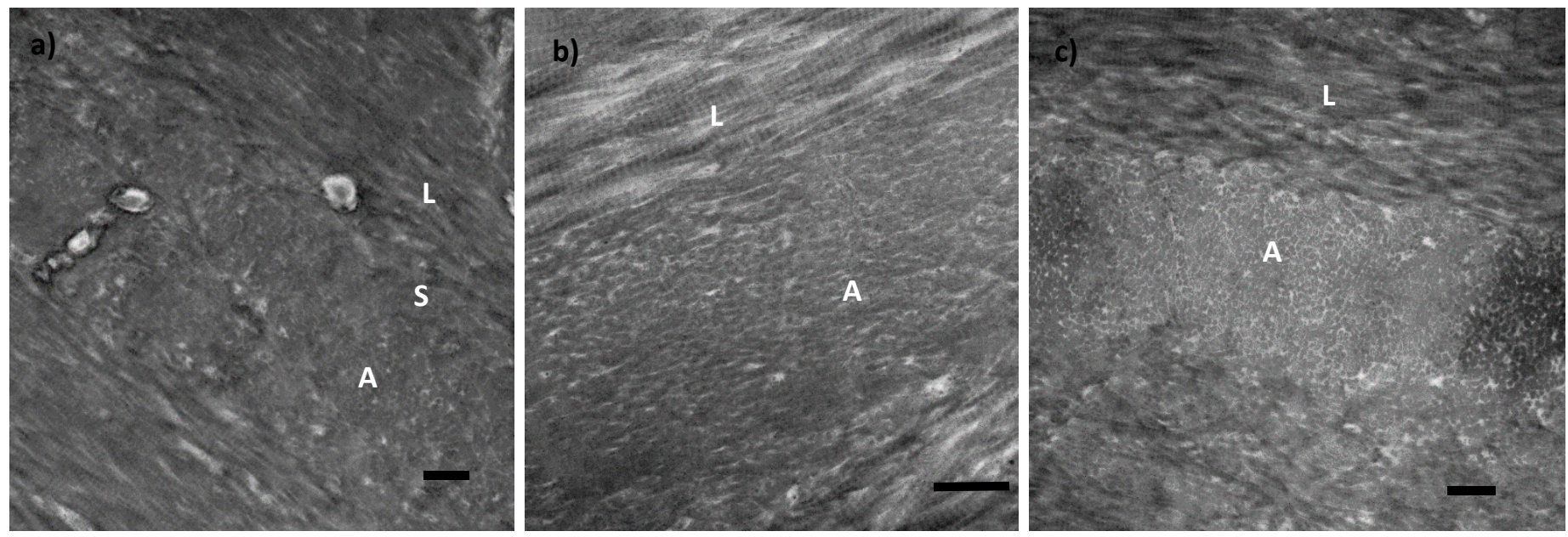

Figure 7: TEM micrographs of trabecular lamellar bone showing the plywood motif within a) Healthy b) Osteolytic (HeLa) and c) Mixed (Ace-1) samples. The plywood motif observed could be twisted in nature showing the characteristic arc pattern due to a successive transition from longitudinal (L), slanted (S) to axial (A) cross-sections (such as in a)) or could be orthogonal in nature with direct transition from longitudinal (L) to axial (A) (such as in b) and c)). Scale bar 500nm.
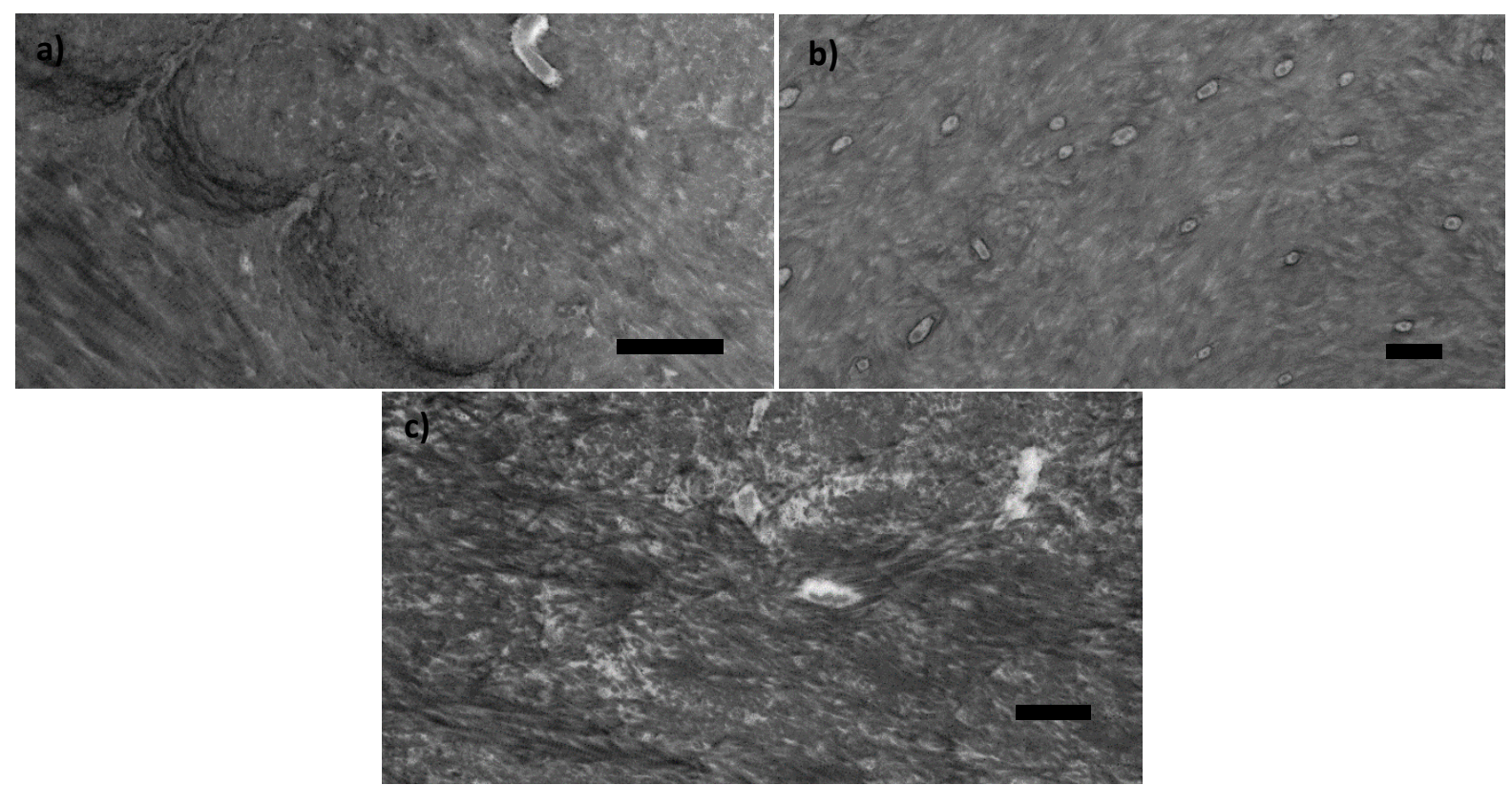

Figure 8: TEM micrographs of trabecular lamellar bone showing deviations from the plywood motif in lamellar bone within a) Healthy b) Osteolytic (HeLa) and c) Mixed (Ace-1) samples. The frequency of such deviations increased in lamellar bone adjacent to woven bone or cartilage (sign of incomplete endochrondral ossification within the trabeculae) and seemed more pronounced in tumour bearing samples. Scale bar $1 \mu \mathrm{m}$. 

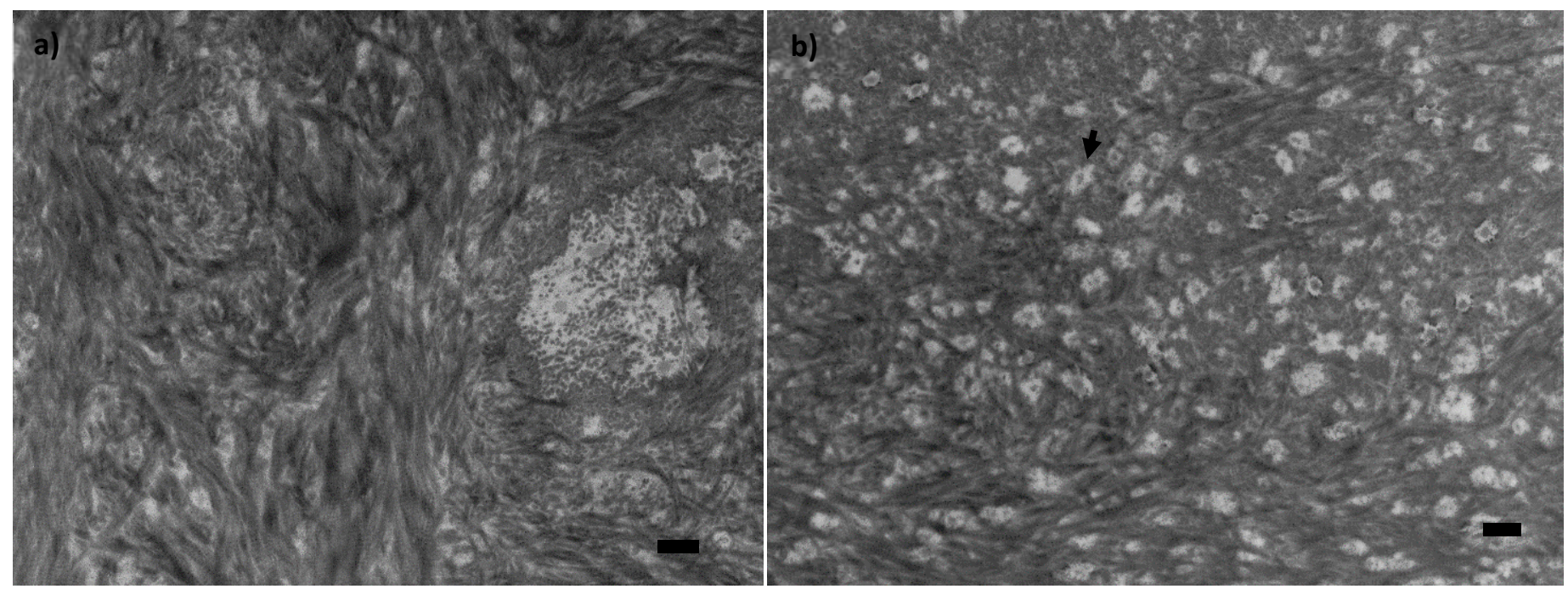

Figure 9: TEM micrographs of a) Pathological new bone formation due to osteoblastic lesions in Ace-1 samples showing high levels of fibril disorganization. b) Woven bone due to incomplete endochrondral ossification within healthy samples. Healthy woven bone shows more organization and higher concentration of canaliculi (highlighted with arrow) Scale bar $500 \mathrm{~nm}$.
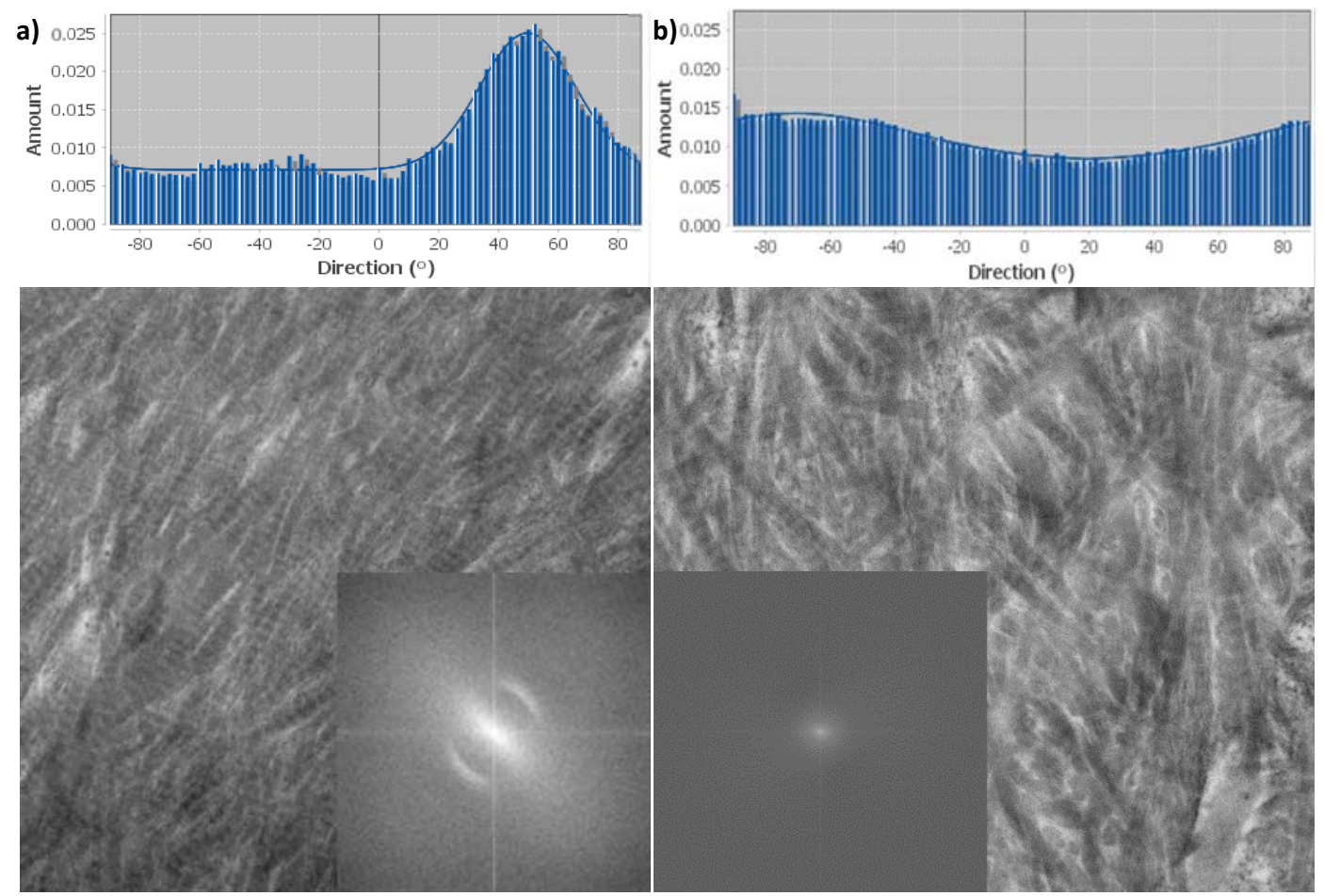

Figure 10: Representative analysis of the degree of disorder of longitudinal orientated collagen fibrils within trabecular bone. The bottom corner inset image is a fast fourier transform of the image showing fibril orientation with respect to micrograph axes. ImageJ/FIJIs directionality plugin produced a histogram distribution of structure orientation from which the degree of disorder could be inferred (top inset image). (a) Parallel versus (b) lack of parallel alignment of collagen fibrils. Scale bar $500 \mathrm{~nm}$. 


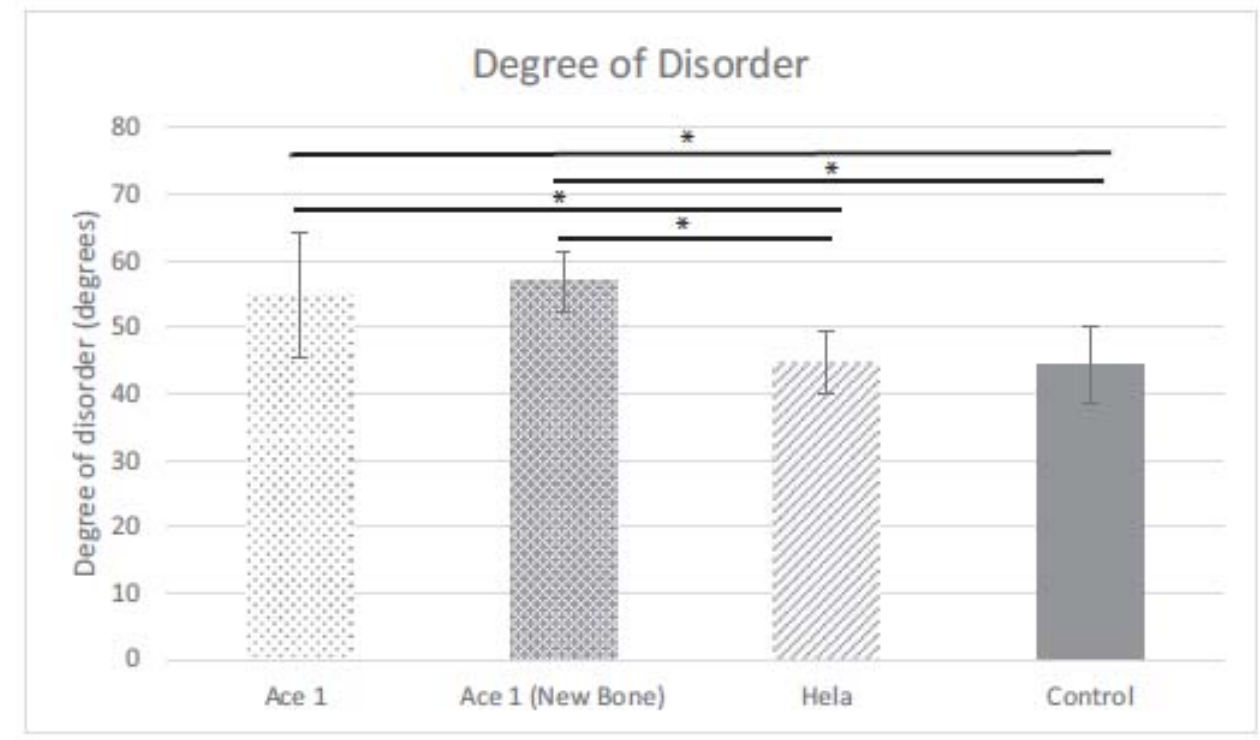

Figure 11: a) Graph showing observed degree of disorder within longitudinal cross-sectioned collagen fibrils within bone of each sample group. Ace-1 bone (both lamellar and pathologic new) showed a significant increase in the degree of disorder compared to healthy control ( $p<0.05)$. There was no significant difference between bone in HeLa-inoculated specimens and healthy controls. 


\begin{tabular}{|c|c|c|}
\hline \multicolumn{3}{|c|}{$\begin{array}{l}\text { a) Factor: Adjacent to Tumour } \\
\text { Dependent Variable: Susceptibility Ratio (R) }\end{array}$} \\
\hline Next to Tumour & Mean & Std. Deviation \\
\hline No & 2.299 & 0.073 \\
\hline Yes & $2.346^{*}$ & 0.082 \\
\hline \multicolumn{3}{|c|}{ p $<0.05$ compared to bone adjacent healthy marrow } \\
\hline \multicolumn{3}{|c|}{ b) Factor: Metastatic Involvement } \\
\hline \multicolumn{3}{|c|}{ Dependent Variable: Susceptibility Ratio (R) } \\
\hline Metastatic Involvement & Mean & Std. Deviation \\
\hline Ace-1 & 2.334 & 0.062 \\
\hline Hela & 2.329 & 0.085 \\
\hline Heal thy & 2.293 & 0.085 \\
\hline \multirow{2}{*}{\multicolumn{3}{|c|}{$\begin{array}{l}\text { c) Factor: Bone Tissue State } \\
\text { Dependent Variable: Susceptibility Ratio (R) }\end{array}$}} \\
\hline & & \\
\hline Bone Tissue Type & Mean & Std. Deviation \\
\hline L. & $2.304^{*}$ & 0.094 \\
\hline 10 & $2.326^{*}$ & 0.060 \\
\hline New & $2.392^{\circ}$ & 0.059 \\
\hline \multicolumn{3}{|c|}{$\mathrm{p}<0.05$ compared to every other type } \\
\hline
\end{tabular}

\begin{abstract}
Table 1: Results of susceptibility ratio measurements $\left(\chi_{\mathrm{ZZZ}}^{(2)^{\prime}} / \chi_{\mathrm{ZXX}}^{(2)^{\prime}}\right)$ with each sample group. (a) Susceptibility ratio between various bone tissue states (fully laminar, incompletely ossified and pathological new bone) were significantly different. (b) Susceptibility ratio between metastatic involvements groups showed no significant difference compared to healthy controls globally however a significant difference was observed between (c) tumour adjacent bone and bone adjacent to healthy marrow.
\end{abstract}

\begin{tabular}{lll}
\hline Sample Group & Average & Std Deviation \\
\hline Ace-1 $(\mathrm{N}=3)$ & 54.78 & 0.95 \\
Ace-1 $(\mathrm{New}$ Bone $)(\mathrm{N}=2)$ & 55.23 & 2.01 \\
HeLa $(\mathrm{N}=5)$ & 54.19 & 2.51 \\
Healthy $(\mathrm{N}=5)$ & 52.30 & 1.19 \\
\hline
\end{tabular}

Table 2: Average fibril diameter measured within bone of each sample group. Although the mean fibril diameter of healthy bone was slightly smaller, there was no significant difference between the groups. 